\title{
Silver-Coated Teflon Tubes for Waveguiding at 1-2 THz
}

\author{
Miguel Navarro-Cía ${ }^{1,2,3,4}$ - Jeffrey E. Melzer ${ }^{5}$. \\ James A. Harrington ${ }^{5}$ Oleg Mitrofanov ${ }^{4}$
}

Received: 20 February 2015 / Accepted: 19 March 2015 /

Published online: 15 April 2015

(C) The Author(s) 2015. This article is published with open access at Springerlink.com

\begin{abstract}
Realization of single-mode low-loss waveguides for 1.0-2.0 THz remains a challenging problem due to large absorption in most dielectrics and ohmic losses in metals. To address this problem, we investigate dielectric-lined hollow metallic waveguides fabricated by coating 1-mm diameter 38- $\mu$ m-thick polytetrafluoroethylene tubes with silver. These waveguides support a hybrid $\mathrm{HE}_{11}$ mode, which exhibits low attenuation and low dispersion. Quasisingle-mode propagation is achieved in the band of 1.0-1.6 THz, in which the hybrid $\mathrm{HE}_{11}$ mode is supported by the waveguide. In this band, the experimentally measured loss is $\sim 20 \mathrm{~dB} / \mathrm{m}\left(\sim 0.046 \mathrm{~cm}^{-1}\right)$, whereas the numerically computed loss is $\sim 7 \mathrm{~dB} / \mathrm{m}$ $\left(\sim 0.016 \mathrm{~cm}^{-1}\right)$. The difference is attributed to additional losses in the dielectric layer, which can be reduced by using alternative polymers.
\end{abstract}

Keywords Near-field time-domain microscopy · Terahertz imaging · Terahertz spectroscopy Terahertz waveguide · Ultrafast measurement

\section{Introduction}

Dielectric-lined hollow metallic waveguides (HMWs) have become a strong candidate for effective single-mode terahertz $(\mathrm{THz})$ propagation with achievable transmission loss below $1 \mathrm{~dB} / \mathrm{m}[1]$. They have been investigated and successfully realized for the frequency band of

Miguel Navarro-Cía

m.navarro@imperial.ac.uk

1 Optical and Semiconductor Devices Group, Department of Electrical and Electronic Engineering, Imperial College London, London SW7 2AZ, UK

2 Centre for Plasmonics and Metamaterials, Imperial College London, London SW7 2AZ, UK

3 Centre for Terahertz Science and Engineering, Imperial College London, London SW7 2AZ, UK

4 Department of Electronic and Electrical Engineering, University College London, Torrington Place, London WC1E 7JE, UK

5 School of Engineering, Rutgers University, 607 Taylor Road, Piscataway, NJ 08854, USA 
$1.5-5.0 \mathrm{THz}[1-5]$, where propagation in free-space [6] or in open waveguides [7, 8] is highly restricted due to water vapor absorption [9].

The dielectric-lined HMWs belong to the family of oversized waveguides [10, 11], which were developed to solve the problem of increasing ohmic losses of single-mode coaxial and rectangular waveguides at millimeter-wave frequencies. By introducing a dielectric coating on the inner metallic walls of an oversized waveguide (as a rule of thumb, $\sim \lambda_{0} / 4$ thick), the waveguide dominant mode becomes the linearly polarized hybrid $\mathrm{HE}_{11}$ mode $[1-5,11-16]$. This $\mathrm{HE}_{11}$ mode is advantageous because it suffers low losses and it couples efficiently to freespace linearly polarized Gaussian beams $[11,17]$. It also can be coupled to the widely used $\mathrm{TE}_{10}$ mode of the rectangular waveguide using a relatively simple conversion [11].

The attenuation of the $\mathrm{HE}_{11}$ mode falls rapidly with the waveguide diameter $[2,3,12$, $18,19]$. The diameter to wavelength ratio $\left(d / \lambda_{0}\right)$ of $\sim 4.4$ or larger (assuming a low-lossdielectric layer) reduces the loss below that of the fundamental $\mathrm{TE}_{11}$ mode of a silver-only waveguide [18]. For dielectrics with losses, the required diameter to wavelength ratio increases.

Despite the oversized waveguide cross-section, the multimode nature is suppressed in dielectric-lined HMWs as the other modes, e.g., the $\mathrm{TE}_{11}, \mathrm{TM}_{11}$, and $\mathrm{TE}_{12}$ modes, suffer a significant increase in their attenuation coefficient. This effect is known as self-filtering [11]. The dielectric-lined HMWs as a result can enable quasi-single-mode propagation with transmission loss potentially lower than that of the fundamental modes in the rectangular waveguide $\left(\mathrm{TE}_{10}\right)$ and in the cylindrical metallic waveguide $\left(\mathrm{TE}_{11}\right)$ with the fractional bandwidth larger than $50 \%$.

To realize a polymer-lined HMW with transmission losses $<10 \mathrm{~dB} / \mathrm{m}$ and self-filtering properties for the $0.5-1.5 \mathrm{THz}$ range, the polymer thickness must be between $\sim 30$ and $\sim 120 \mu \mathrm{m}$ and $d>0.9 \mathrm{~mm}$. Depositing uniform polymer layers of such thickness remains a challenge for the dynamic liquid phase fabrication technique [20] used for the $1.5-5.0 \mathrm{THz}$ range. Alternative methods, such as mandrel-winding [5], mechanical-assembling of dielectric and metallic cylinders [11, 13, 14], and high-temperature bonding [21] do not offer a solution because the minimum diameter $d$ for these methods is limited to $\sim 3 \mathrm{~mm}$, which allows undesirable higher-order modes, and fabrication tolerances are excessive.

A way to circumvent the obstacle of fabricating dielectric-lined HMWs for $0.5 \mathrm{THz}<v<1.5$ $\mathrm{THz}$ may be found by metallizing the outer surface of polymer tubes [19], which are cheaply and easily available in a wide range of thicknesses and diameters. Among the large catalogue of polymer tubes, those made of polytetrafluoroethylene (PTFE), commonly known by its trade name Teflon, may be good candidates. PTFE was indeed used for the original millimeterwave dielectric-lined HMWs [11] and it is widely used for many millimeter-wave devices because of its low absorption $[22,23]$. Although the absorption of PTFE is not the lowest among polymers and it increases with frequency, this material can serve to test properties of the waveguides fabricated by this method and to establish a benchmark for future investigations with low-loss polymers.

To answer the question whether commercial PTFE tubes are suitable for fabrication of the high-performance dielectric-lined HMW for $0.5 \mathrm{THz}<v<1.5 \mathrm{THz}$ band, we characterize transmission of THz pulses through PTFE tubes coated with silver experimentally. The extracted transmission loss properties are compared with eigenmode simulations.

PTFE tubes of $1 \mathrm{~mm}$ bore diameter and $\sim 38-\mu \mathrm{m}$-thick walls are chosen for this study. According to initial analytical work, the fundamental $\mathrm{HE}_{11}$ mode for a waveguide with such 
dimensions $\left(d / \lambda_{0} \sim 4.4\right)$ should have attenuation $\sim 10 \mathrm{~dB} / \mathrm{m}$, similar to the fundamental $\mathrm{TE}_{11}$ mode of a silver-only waveguide within the transmission band centered at $1.3-1.4 \mathrm{THz}$. The silver-only waveguide with the same diameter however is multimode for the whole spectral window studied here.

We fabricate flexible waveguides by coating the PTFE tube with silver. The quasi-singlemode $\left(\mathrm{HE}_{11}\right)$ propagation is observed after $\sim 283 \mathrm{~mm}$ of propagation in the waveguide. We find the transmission loss of $\sim 20 \mathrm{~dB} / \mathrm{m}$ within a wide transmission band $(\sim 1.0$ to $\sim 1.6 \mathrm{THz})$ experimentally using the cut-back loss method and the THz time-domain spectroscopy system. The numerical simulations show evolution of mode profiles for the supported modes and their attenuation with frequency, and explain the quasi-single-mode $\left(\mathrm{HE}_{11}\right)$ propagation. Comparison of the experimental and simulation results suggests that dielectric losses in PTFE used in this study are larger than nominal. Nevertheless, this waveguide concept realized with other lower-loss dielectric tube can lead to quasi-single-mode waveguides outperforming cylindrical metallic waveguides of similar diameter.

\section{Fabrication and experimental setup}

\subsection{Fabrication}

The silver-coated PTFE hollow waveguide is fabricated with a three-step procedure [19]. First, the outer surface of the 1-mm-bore diameter flexible PTFE tube (Zeus Inc. [24]) is etched using a commercial etchant, FluorEtch $($, from Acton Technologies. As the surface energy of PTFE is exceptionally low, this step is necessary to ensure subsequent adhesion of the silver film. Second, a palladium-based sensitizing solution is used to further treat the tube surface. Finally, the silver layer is formed via dynamic liquid phase deposition of ammonia-complexed silver ion and dextrose solutions.

Thickness of the PTFE layer is verified using the transmission spectrum of the waveguide in the infrared region. Figure 1 shows the spectrum obtained using a Tensor Bruker 37 FTIR spectrometer and liquid-nitrogen-cooled mercury cadmium telluride detector in the 5.5-7 $\mu \mathrm{m}$ range for a $283-\mathrm{mm}$ waveguide. Taking the average interference peak spacing and assuming a refractive index of 1.35 , the film thickness is determined to be approximately $35 \mu \mathrm{m}$, close to the expected thickness of $38 \mu \mathrm{m}$ [19].

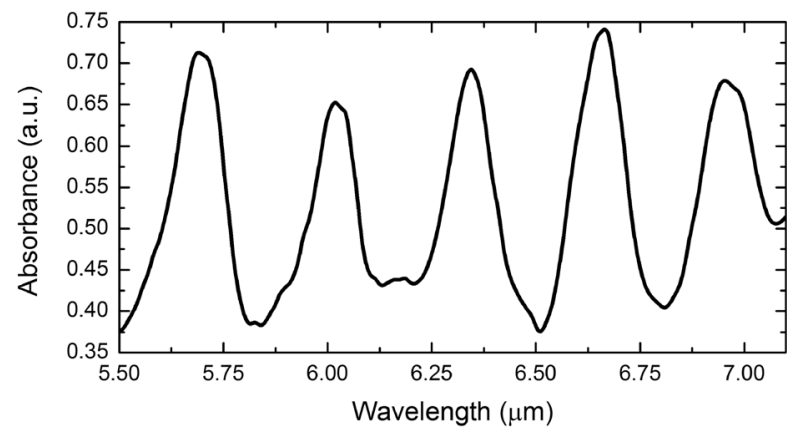

Fig. 1 Infrared spectrum of the $283 \mathrm{~mm}$ waveguide obtained with a Tensor Bruker 37 FTIR spectrometer 


\subsection{Experimental setup}

We characterize the waveguide samples using the near-field $\mathrm{THz}$ time-domain spectroscopy method (shown in Section 4). Near-field mapping of the terahertz field at the waveguide output allows us to optimize coupling from the incident terahertz pulse and determine the spatial mode profiles. The cut-back loss measurement method also avoids potential errors due to the variation of the coupling coefficient with frequency $[4,25,26]$.

The waveguide part of the near-field mode spectroscopy and imaging system $[4,27,28]$ is shown in detail in Fig. 2a-c. THz pulses are generated by horizontally polarized $\left(E_{x}\right) 100 \mathrm{fs}$ pulses from a Ti:Sapphire mode-locked laser through optical rectification in a 1-mm-thick $\mathrm{ZnTe}$ crystal. The generated $E_{x}$-polarized $\mathrm{THz}$ beam is then collimated by a 3-mm-diameter hyper hemispherical lens. The waveguide sample is placed at a distance of $\sim 500 \mu \mathrm{m}$ away from the lens, see Fig. $2 b$. The output of the waveguide is mounted on an automated $x y$ translation stage for mapping the waveguide modes and it is positioned within $\sim 1 \mathrm{~mm}$ from the integrated terahertz near-field probe, as shown in Fig. 2c. The near-field probe consists of a low-temperature-grown GaAs photoconductive detector sensitive to the horizontal polarization, $E_{x}$, and a $10 \mu \mathrm{m} \times 10 \mu \mathrm{m}$ sub-wavelength aperture [26, 29]. In order to optimize coupling of the incident terahertz beam to the fundamental hybrid waveguide mode and to minimize the excitation of higher-order modes, the $x y$-position of the input waveguide facet is adjusted while monitoring the output field pattern. The input and the output of the waveguide are enclosed
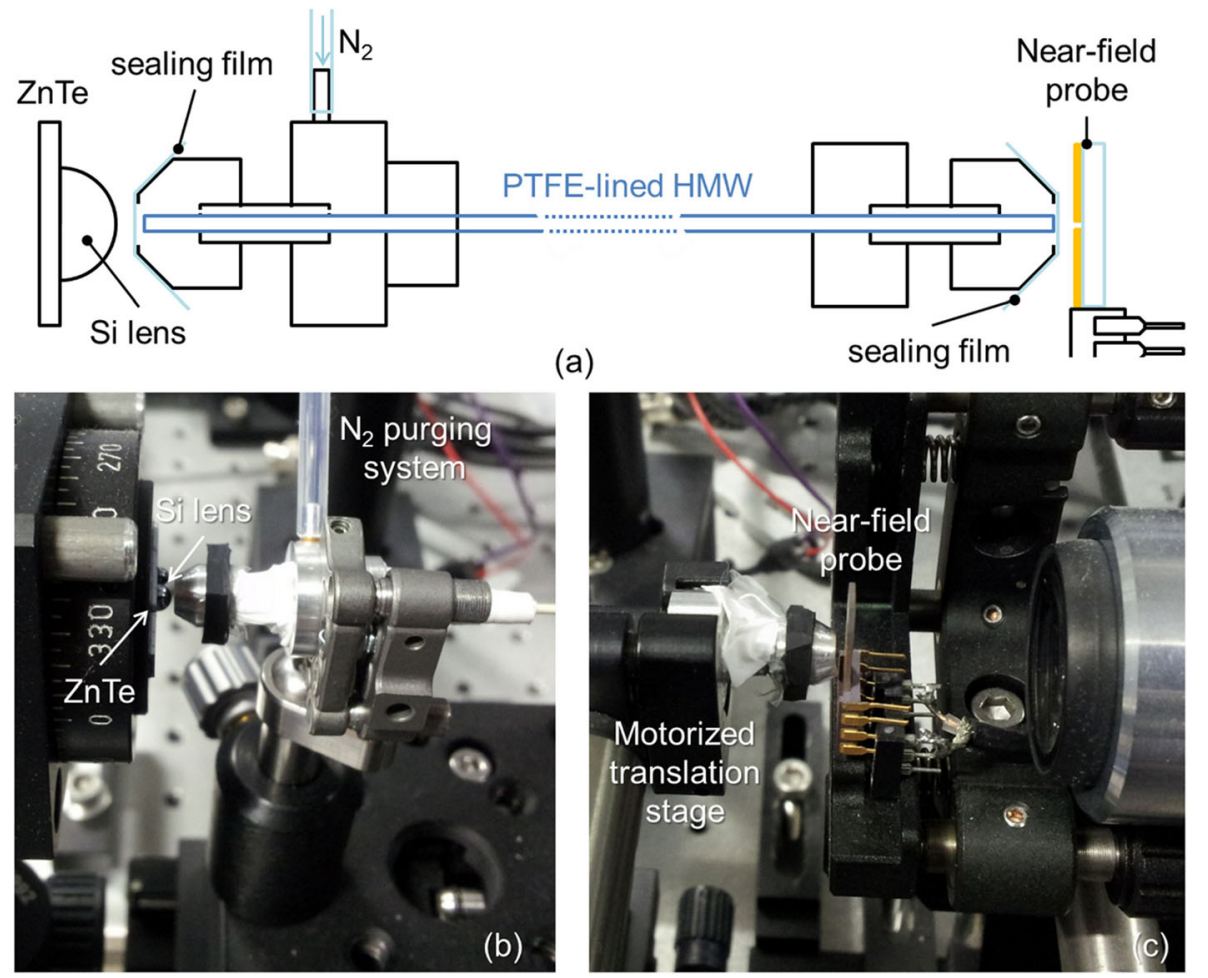

Fig. 2 a Sketch of the setup along with the description of the different elements. b Detail of the input. c Detail of the output section 
into sealed holders and purged with $\mathrm{N}_{2}$ to reduce water vapor absorption inside the waveguide (see Fig. 2a, b for the $\mathrm{N}_{2}$ inlet, and Fig. 2a, c for the output holder).

\section{Numerical modeling}

We first review the eigenmodes supported by the PTFE-lined HMW to provide a basis for the analysis of the experimental results.

\subsection{Transmission losses and field distribution inside the waveguide}

In order to model the experiment accurately, we calculate the eigenmodes and the corresponding eigenvalues (dispersion diagram) of the PTFE-lined HMW numerically with the 2D eigenmode solver of CST Microwave Studio ${ }^{\mathrm{TM}}$. The effective dispersive silver conductivity model and two sets of absorption data for PTFE are used (Fig. 3a). The first set represents tabulated dielectric properties of PTFE $[22,23]$. In the second set, we assume that the imaginary values of the tabulated data are increased by a factor of 4 . We note that such absorption above the nominal PTFE absorption is comparable to the absorption values reported for other polymers [30,31]. A detailed description of the simulation can be found in Appendix: Eigenmode numerical analysis.

The attenuation coefficient curves for the first four modes that are most likely to be excited under the on-center illumination in our experimental system are shown in Fig. 3b. Electric field profiles of these modes are shown in Fig. 4. At very low frequencies, the PTFE film is electrically negligible, and thus, the PTFE-lined HMW supports similar modes as a silver-only HMW [25, 32-34]. Hence, we denote the discussed modes in the case of the PTFE-lined HMW as $\mathrm{TE}_{11}{ }^{*}, \mathrm{TM}_{11}{ }^{*}, \mathrm{TE}_{12}{ }^{*}$, and $\mathrm{TM}_{12}{ }^{*}$.

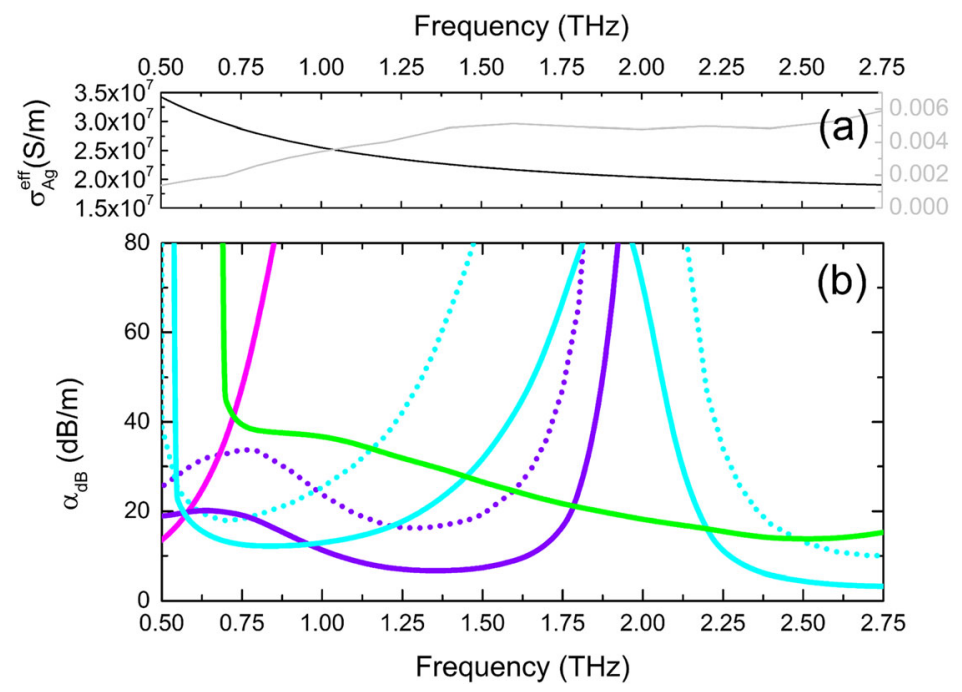

Fig. 3 a Material properties [the real part of the PTFE permittivity is $\varepsilon=2.074]$. b Numerically computed attenuation for the fundamental modes: first (magenta), second (violet), third (cyan), and fourth (green); solid and dotted lines account for nominal PTFE according to tabulated data $\varepsilon$ and the lossy PTFE with $4 \varepsilon$, respectively 


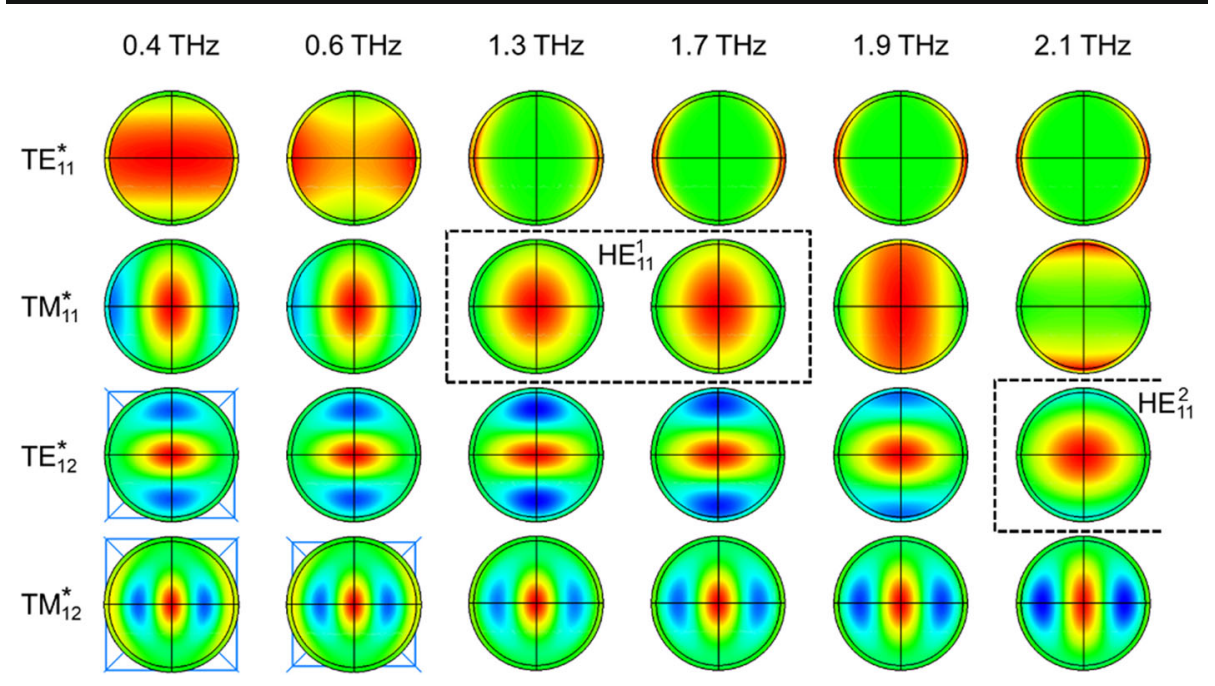

Fig. $4 E_{x}(x, y)$ for the $\mathrm{TE}_{11}{ }^{*}$ (first row), $\mathrm{TM}_{11}{ }^{*} \rightarrow \mathrm{HE}_{11}{ }^{1}$ (second row), $\mathrm{TE}_{12}{ }^{*} \rightarrow \mathrm{HE}_{11}{ }^{2}$ (third row), and $\mathrm{TM}_{12}{ }^{*}$ mode (fourth row) at, from left to right, $0.4,0.6,1.3,1.7,1.9$, and $2.1 \mathrm{THz}$. The external blue lines in the bottom left corner highlight that the mode is in cut-off for such frequency. The hybrid $\mathrm{HE}_{11}$ modes are explicitly underlined with dashed black lines

Attenuation of the $\mathrm{TE}_{11}{ }^{*}$ mode, which has the lowest cut-off frequency mode (magenta line in Fig. 3b), increases drastically with frequency. Near its cut-off frequency, the mode profile is identical to the $\mathrm{TE}_{11}$ mode of the HMW. At high frequencies, the $\mathrm{TE}_{11}{ }^{*}$ mode evolves into a field distribution highly localized in the PTFE layer (Fig. 4), resulting into a fast increase in its attenuation coefficient (Fig. 3b) [34]. Above $0.75 \mathrm{THz}$, the higher-order modes show significantly lower attenuation.

The second mode $\left(\mathrm{TM}_{11}{ }^{*}\right.$, violet line in Fig. $\left.3 \mathrm{~b}\right)$ suffers some increase in attenuation within the lowest part of the spectral window. However, at $\sim 0.75 \mathrm{THz}$ there is an inflection point and the attenuation drops, despite the increase in material absorption (Fig. 3a). A transmission band is formed for this mode within $0.9-1.7 \mathrm{THz}$ with the minimum attenuation of $\sim 6.6 \mathrm{~dB} / \mathrm{m}$ $\left(\sim 0.015 \mathrm{~cm}^{-1}\right)$. Within this band, waves travel as the $\mathrm{HE}_{11}{ }^{1}$ mode (Fig. 4), where the superscript denotes the order of the transmission band. The hybrid $\mathrm{HE}_{11}{ }^{1}$ mode has negligible field at the walls, and therefore the dielectric and conductive losses are minimized. We note that the other modes have significantly higher attenuation within this frequency range, i.e., self-filtering is expected. For $\nu>1.7 \mathrm{THz}$, the attenuation of this second mode suddenly rises as a result of field concentration in the top and bottom air-dielectric interface (Fig. 4).

The third mode $\left(\mathrm{TE}_{12}{ }^{*}\right.$, cyan line in Fig $3 \mathrm{~b}$ ) has two distinct transmission bands: $0.6 \mathrm{THz}$ $<\nu<1.2 \mathrm{THz}$ and for $\nu>2.2 \mathrm{THz}$. The $\mathrm{TE}_{12}{ }^{*}$ mode maintains the field distribution almost uniformly until the absorption band centered at $\sim 1.9 \mathrm{THz}$. Hence, its attenuation coefficient does not show any sudden change at low frequencies. In the second band, the mode transforms into the $\mathrm{HE}_{11}$-like mode (Fig. 4). To differentiate it from the lower-frequency $\mathrm{HE}_{11}$ mode, we will use the following notation: $\mathrm{HE}_{11}{ }^{2}$.

Finally, the attenuation of the fourth mode $\left(\mathrm{TM}_{12}{ }^{*}\right.$, green line in Fig. 3b) decreases gradually with frequency, displaying relatively low attenuation for $\nu>2.2 \mathrm{THz}$. The field distribution remains almost constant, which explains the gradual changes observed in its attenuation coefficient. 
For both hybrid $\mathrm{HE}_{11}$ modes, the transmission bands become narrower and the attenuation increases when higher dielectric losses are considered in the modeling [4, 18]. Specifically, the $\mathrm{HE}_{11}{ }^{1}$ mode transmission band is limited between 1.0 and $1.6 \mathrm{THz}$ with a minimum attenuation of $\sim 16.2 \mathrm{~dB} / \mathrm{m}\left(\sim 0.037 \mathrm{~cm}^{-1}\right)$.

\subsection{Impact of bending and metal model in transmission losses}

We investigate robustness of the effective single-mode $\mathrm{HE}_{11}{ }^{1}$ propagation against bending by computing the total attenuation as a function of the bending radii with Lumerical MODE Solutions (further details can be found in Appendix: Eigenmode numerical analysis). Losses due to bending in the plane parallel to the polarization $(x z)$ are shown in Fig. 5a, and in the plane perpendicular to the polarization $(y z)$ in Fig. $5 \mathrm{~b}$ for the $\mathrm{HE}_{11}{ }^{1}, \mathrm{TE}_{12}{ }^{*}$, and $\mathrm{TM}_{12}{ }^{*}$ modes. Although the total attenuation follows different trends for each mode, it is evident that the effective single-mode $\mathrm{HE}_{11}{ }^{1}$ propagation endures within the spectral window of interest (1.0$1.6 \mathrm{THz}$ ) for bending radii larger than $25 \mathrm{~mm}$.

We estimate the effect of metallic losses within the transmission band numerically by considering four models for the metallic walls. The minimum loss value of $3.2 \mathrm{~dB} / \mathrm{m}$ is found for perfect electric conductor, $5.3 \mathrm{~dB} / \mathrm{m}$ for bulk silver $\left(\sigma_{\mathrm{Ag}}=6.3 \times 10^{7} \mathrm{~S} / \mathrm{m}\right)$ and 6.6 and $7.0 \mathrm{~dB} / \mathrm{m}$ for silver with surface roughness of 60 and $100 \mathrm{~nm}$ (RMS), respectively. These results indicate that surface roughness has a small effect on the overall transmission loss.

\subsection{Dispersion: modal group delay}

The numerically computed eigenvalues allow us to construct the expected modal group delay for the two sample lengths used in this study: 109 and $283 \mathrm{~mm}$. These results are plotted in Figs. $6 \mathrm{a}$ and $7 \mathrm{a}$, where the absorption band is clearly marked as a gray rectangular box.

By inspection of Figs. $6 \mathrm{a}$ and $7 \mathrm{a}$, it is evident that the high-frequency $\mathrm{HE}_{11}{ }^{2}$ mode arrives earlier than the $\mathrm{HE}_{11}{ }^{1}$ mode. According to the numerically computed group delays, the hybrid $\mathrm{HE}_{11}{ }^{2}$ mode is the only mode present with the spectral components above $2.2 \mathrm{THz}$ for $t<5.5 \mathrm{ps}$ in the launch waveguide and for $t<17.9 \mathrm{ps}$ in the $283 \mathrm{~mm}$ long waveguide. Within the intervals
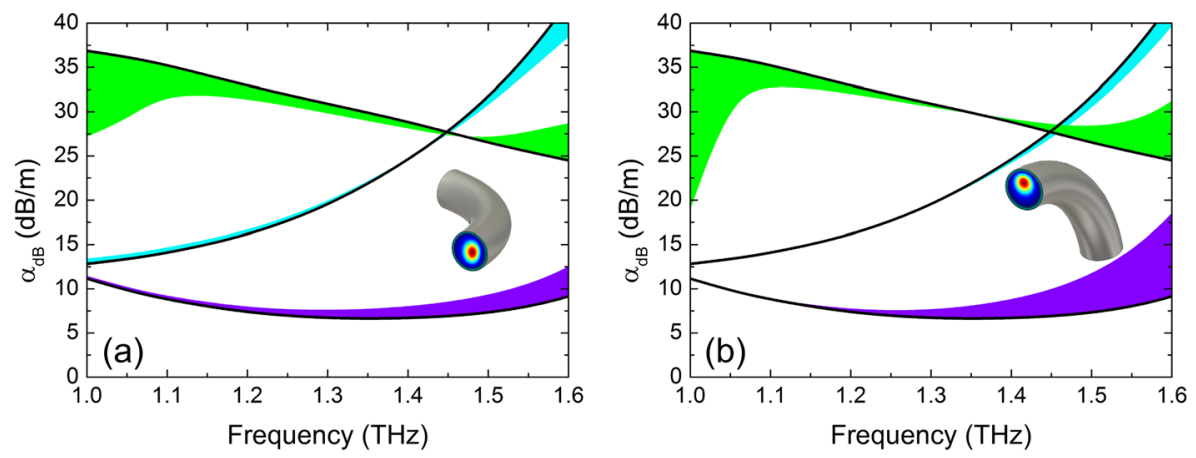

Fig. 5 Numerically computed attenuation for the second (violet), third (cyan), and fourth (green) modes for bending radii from infinite (i.e., straight waveguide) to $25 \mathrm{~mm}$ : a $x z$-plane of bending, and $\mathbf{b} y z$ plane of bending. To discern the evolution of the attenuation with the bending radii, the modal loss for the straight waveguide is shown by solid black lines. Insets in each panel show the bent waveguide along with the $\mathrm{HE}_{11}(\nu=1.3 \mathrm{THz})$ mode profile for a bend radius of $25 \mathrm{~mm}$ 

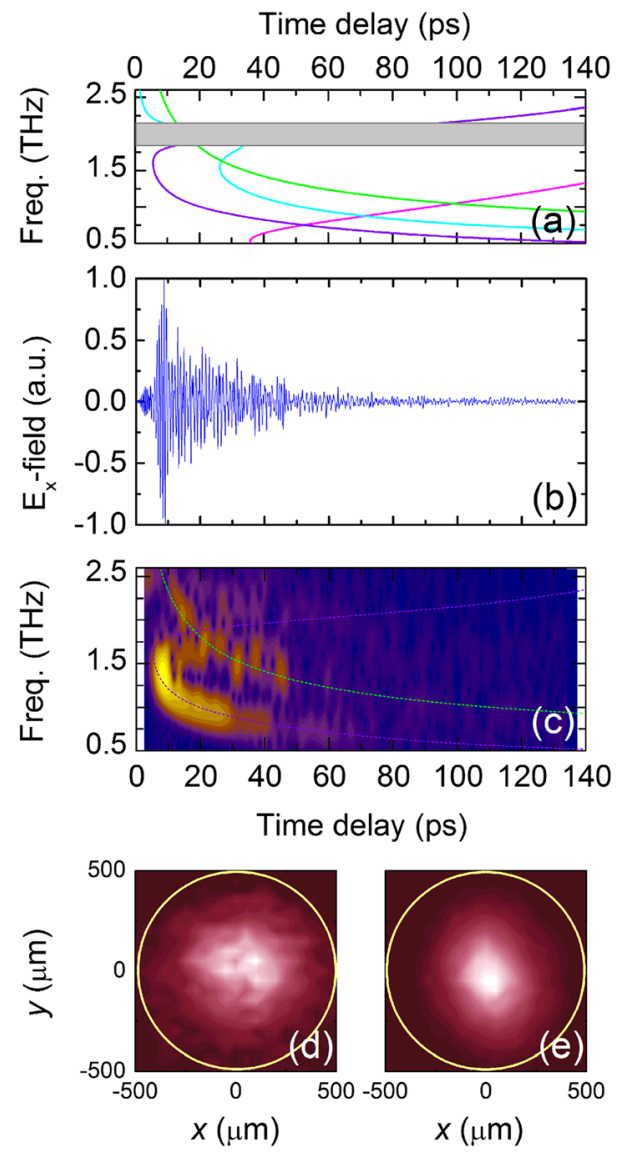

Fig. 6 a Numerically computed group delay for the $\mathrm{TE}_{11}{ }^{*}$ (magenta), $\mathrm{TM}_{11}{ }^{*}$ (violet), $\mathrm{TE}_{12}{ }^{*}$ (cyan), and $\mathrm{TM}_{12}{ }^{*}$ (green) modes in the launch waveguide. The gray rectangle represents the absorption band that defines the border between the $\mathrm{HE}_{11}{ }^{1}$ and the $\mathrm{HE}_{11}^{2}$ mode band. b Waveform detected on the waveguide axis. c Power spectral density of the measured waveform shown right above $\mathbf{b}$. The numerically computed group delay for the $\mathrm{TM}_{11}{ }^{*}$ (dotted violet) and the $\mathrm{TM}_{12}{ }^{*}$ modes (dotted green) are superimposed to facilitate the comparison. d, e Normalized near-field $\left|E_{x}\right|^{2}$ profiles at the waveguide output at 2.73 (d) and 8.73 ps (e). The yellow circle outlines the waveguide walls to guide the eye

5.5-10 ps and 18-27 ps for the two waveguides, respectively, the $\mathrm{HE}_{11}{ }^{1}$ mode is the only mode present. At later times, the $\mathrm{TM}_{12}{ }^{*}$ mode (green line) with frequency components above $2 \mathrm{THz}$ is also present. Since this TM mode has relative low loss at high frequencies, signs of multimode propagation may be expected experimentally in the waveforms for time delays beyond $\sim 10$ and $\sim 27$ ps for the two waveguides, respectively. In frequency domain, we then expect spectra relatively clear of mode interference peaks within the transmission band 1.0-1.6 THz. Although in principle multimode propagation is expected within this band, the large attenuation of the $\mathrm{TE}_{12}{ }^{*}$ and $\mathrm{TM}_{12}{ }^{*}$ modes results in quasi-single-mode $\left(\mathrm{HE}_{11}{ }^{1}\right)$ propagation.

The comparison of the modal group delay for each length exposes an issue for the calculation of the transmission spectrum. For the longer waveguide, the higher-order $\mathrm{TE}_{12}{ }^{*}$ mode (cyan line) arrives after the measured interval of $140 \mathrm{ps}$ for $\nu<1.0 \mathrm{THz}$. The recorded waveform therefore is missing the frequency components from 0.75 to $1.0 \mathrm{THz}$ for the 283 - 
Time delay (ps)
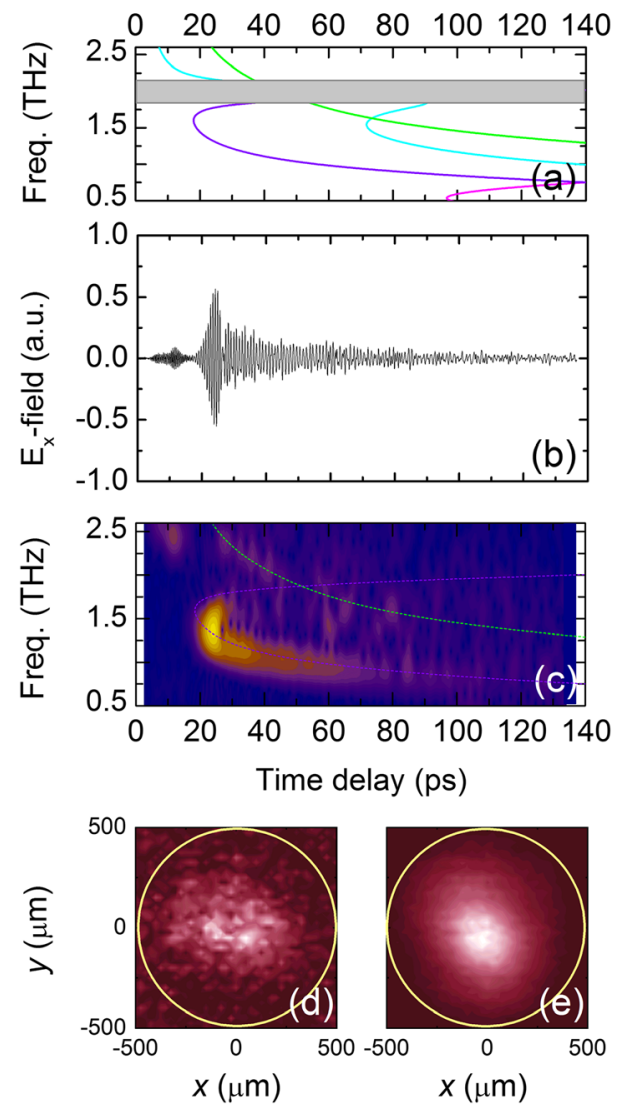

Fig. 7 a Numerically computed group delay for the $\mathrm{TE}_{11}{ }^{*}$ (magenta), $\mathrm{TM}_{11}{ }^{*}$ (violet), $\mathrm{TE}_{12}{ }^{*}$ (cyan), and $\mathrm{TM}_{12}{ }^{*}$ (green) modes in the $283 \mathrm{~mm}$ long waveguide. The gray rectangle represents the absorption band that defines the border between the $\mathrm{HE}_{11}{ }^{1}$ and the $\mathrm{HE}_{11}{ }^{2}$ mode band. b Waveform detected on the waveguide axis. c Power spectral density of the measured waveform shown right above $\mathbf{b}$. The numerically computed group delay for the $\mathrm{TM}_{11}{ }^{*}$ (dotted violet) and the $\mathrm{TM}_{12}{ }^{*}$ modes (dotted green) are superimposed to facilitate the comparison. d, $\mathbf{e}$ Normalized near-field $\left|E_{x}\right|^{2}$ profiles at the waveguide output at 11.73 (d) and $24.6 \mathrm{ps}(\mathbf{e})$. The yellow circle outlines the waveguide walls to guide the eye

mm-long waveguide. If the input terahertz beam is coupled initially to the $\mathrm{TM}_{11}{ }^{*}$ mode and the $\mathrm{TE}_{12}{ }^{*}$ mode, the calculation of the transmission spectrum overestimates losses within the $0.75-1.0 \mathrm{THz}$ frequency span. Indeed, excitation of both modes is likely at low frequencies since both modes have similar overlap integrals with the Gaussian beam. A similar argument is applied to $\nu<0.75 \mathrm{THz}$, for the $\mathrm{TE}_{11}{ }^{*}$ and $\mathrm{TM}_{12}{ }^{*}$ modes. Therefore, for $\nu<1.0 \mathrm{THz}$, the experimentally measured losses can only provide an upper bound.

\section{Near-field time-domain measurements and discussion}

The output waveform and $E$-field $x y$-maps are measured for the launch waveguide followed by the same set of experiments for the 283-mm-long waveguide, which is constructed by 
attaching an additional segment of $174 \mathrm{~mm}$ to the launch waveguide. This approach allows us to determine the transmission loss produced by the 174-mm segment.

The waveforms detected in the center of the waveguide output for the launch and joined samples are shown in Figs. 6b and 7b, respectively. Two pulses can be resolved in both waveforms. Using the modeling results, we could tentatively assign them to the $\mathrm{HE}_{11}{ }^{2}$ and $\mathrm{HE}_{11}{ }^{1}$ modes. Indeed, the intensity $x y$-map at the peaks of the leading and second transmitted pulses reveals $\mathrm{HE}_{11}$ mode profile for both pulses (Figs. 5d, e and 6d, e), confirming the assumption.

Signs of multimode interference are also noticeable for the second pulse. Namely, the coherent oscillations in the pulse are interrupted at $\sim 10$ and $\sim 27 \mathrm{ps}$ for the launch and 283-mmlong waveguide, respectively. This is also in agreement with the numerical results.

The mode interference can be observed in the spectrograms, i.e., time-frequency-maps, computed for each waveform (Figs. $6 \mathrm{c}$ and $7 \mathrm{c}$ ). The waveform is divided into 28 Hammingwindowed segments with $90 \%$ overlap. At earlier time delays, only the spectral component at around $2.4 \mathrm{THz}$, which is linked to the $\mathrm{HE}_{11}{ }^{2}$ mode, is observed. The strongest signal is at 1.11.6 THz in both spectrograms. The $\mathrm{HE}_{11}{ }^{1}$ mode is expected in this range. Low dispersion can be seen near the band center. The lack of signal around $1.9 \mathrm{THz}$ is linked to the absorption band.

In both spectrograms, the $\mathrm{HE}_{11}{ }^{1}$ mode (violet line) forms a tail due to large dispersion for $v$ $<1.1 \mathrm{THz}$, when it changes to the $\mathrm{TM}_{11}{ }^{*}$ mode. The $\mathrm{TM}_{12}{ }^{*}$ mode (green line) also yields another tail for $1.3 \mathrm{THz}<v<2.5 \mathrm{THz}$. For the 283 -mm-long waveguide, the trace associated to the $\mathrm{TM}_{12}{ }^{*}$ mode is almost absent, suggesting that quasi-single $\mathrm{HE}_{11}$ mode propagation is obtained after $283 \mathrm{~mm}$ within the $\mathrm{HE}_{11}{ }^{1}$ transmission band.

The waveguide transmission spectrum is calculated by taking the ratio of the power spectra (Fig. 8a). Figure $8 \mathrm{~b}$ presents the attenuation in decibels per meter, $\alpha_{\mathrm{dB}}$. A signal-to-noise ratio above 10 is achieved within a frequency span from 0.5 to $2.75 \mathrm{THz}$ (spectral window highlighted by the orange box in Fig. 8a). This spectral region includes the fundamental transmission band, the absorption band and the low frequency part of the second transmission band $[4,5,19,32-34]$.

In the transmission band, $1.0 \mathrm{THz}<\nu<1.6 \mathrm{THz}$, where the hybrid mode $\mathrm{HE}_{11}$ is fully developed, losses decrease to $\sim 17 \mathrm{~dB} / \mathrm{m}\left(\sim 0.039 \mathrm{~cm}^{-1}\right)$ at approximately $1.3 \mathrm{THz}$. To put this value of attenuation in context, it is worth looking at commercial single-mode hollow waveguides. For instance, the rectangular waveguide WR- 0.65 used by Virginia Diodes Inc. to cover the bandwidth 1.1-1.7 THz (similar to the transmission band here) has an estimated waveguide loss between 258 and $406 \mathrm{~dB} / \mathrm{m}$ at the low and high end of the band, respectively [35], which is hundreds of decibels higher than our experimentally reported waveguide loss. The experimental spectrum is consistent with the prediction that the transmission loss is minimal for this waveguide at $\sim 1-2 \mathrm{THz}$. The spectrum quantitatively matches the transmission band from the simulation with dielectric absorption larger than the nominal used before

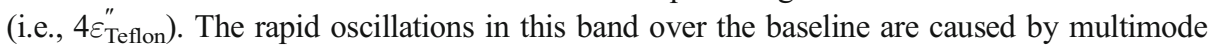
propagation involving the dominant $\mathrm{HE}_{11}{ }^{1}$ and the $\mathrm{TM}_{12}{ }^{*}$ modes.

The more pronounced rapid oscillations observed for $\nu>1.6 \mathrm{THz}$ in both waveform spectra (Fig. 8a) as well as in the transmission spectrum (Fig. 8b) suggest multimode propagation, but with a less defined dominant mode. In this range, the $\mathrm{HE}_{11}$ and $\mathrm{TM}_{12}{ }^{*}$ modes travel with similar attenuation (see Fig. 8b), and thus, there is not a distinct dominant mode producing a clear baseline. This is in agreement with the spectrograms and the modeling. 

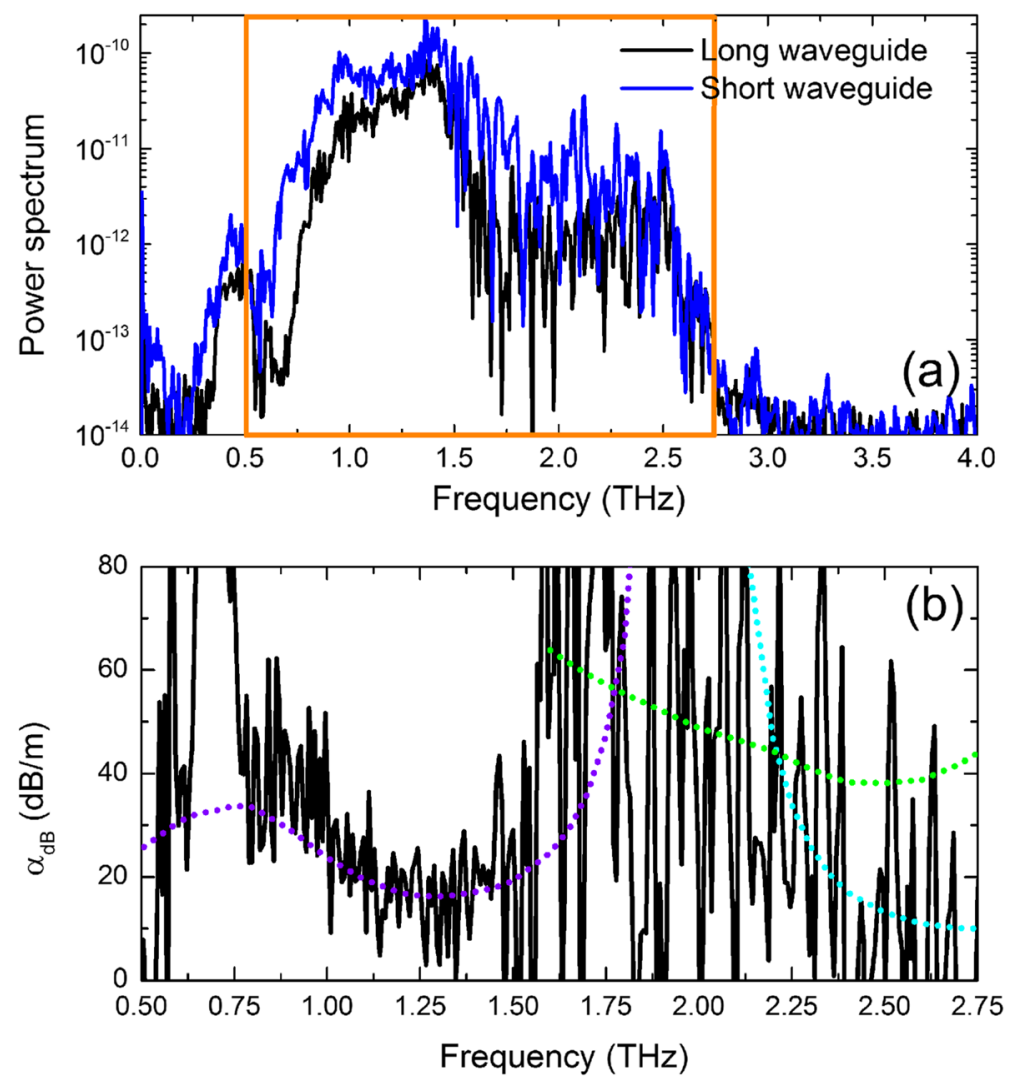

Fig. 8 a Power spectra for the launch (blue) and 283-mm-long waveguide (black); the orange rectangle highlights the spectral window represented in panel b. b Transmission loss measured by the cut-back experiment (black) together with the numerically computed attenuation for the fundamental $\mathrm{TM}_{11}{ }^{*} \rightarrow \mathrm{HE}_{11}{ }^{1}$ (violet),

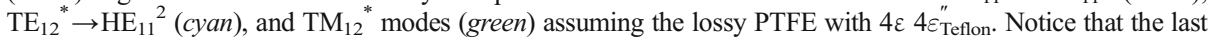
two modes are only displayed within a specific range

\section{Comparison with the literature}

Different fabrication techniques have been used for dielectric-lined HMWs. For $v>0.5 \mathrm{THz}$, mandrel-wiring [5] and dynamic liquid phase deposition [20] can produce waveguides with reduced diameters required for suppressing higher-order modes and for waveguide flexibility. The reported characteristics of such waveguides are summarized in Table 1, and they will be discussed afterwards.

These two techniques, however, have not produced waveguides with $d<3 \mathrm{~mm}$ (Fig. 9). There is still a niche for fabrication dielectric-lined HWMs with radii above the $d / \lambda_{0}=4$ line, where they outperform metal-only waveguides of the same diameter. The results presented here show that the coating of polymer tubes gives access to such restricted area.

Table 1 shows the transmission loss of different terahertz-dielectric-lined HMWs reported in the literature that operate in the spectral window of interest for this paper together with our initial findings with PTFE for the lowest part of the terahertz-gap, i.e., 0.5 THz. 
Table 1 Experimental loss of $\mathrm{THz}$ dielectric-lined hollow metallic waveguides

\begin{tabular}{llccl}
\hline Material & Central Frequency $(\mathrm{THz})$ & $d / \lambda_{0}$ & $\alpha_{\mathrm{dB}}(\mathrm{dB} / \mathrm{m})$ & Ref. \\
\hline $\mathrm{Ag} / \mathrm{PTFE}$ & 0.5 & 3.3 & 7 & {$[19]$} \\
& 1.3 & 4.3 & 17 & This work \\
$\mathrm{Ag} / \mathrm{PS}$ & 1.4 & 18.7 & 1.8 & {$[3]$} \\
$\mathrm{Ag} / \mathrm{PE}$ & 1.5 & 15.0 & 1.3 & {$[5]$} \\
$\mathrm{Ag} / \mathrm{COP}$ & 1.7 & 17.0 & 1.5 & {$[5]$} \\
\hline
\end{tabular}

The attenuation for our previous PTFE-lined HMW was lower than that reported here despite its smaller $d / \lambda_{0}$. This is explained by the significantly lower absorption of PTFE (Fig. 3a) at $\nu<1 \mathrm{THz}$, the higher effective conductivity of Ag and the lower influence of the roughness.

HMWs coated with polystyrene (PS), polyethylene (PE), or cyclic olefin copolymer (COP) have shown very low attenuation when the $d / \lambda_{0}$ is larger than 15 . Specifically, attenuations one order of magnitude lower than the attenuation here reported were measured. This is, in principle, expected given the rapid drop undergone by the attenuation with the diameter size [2, 3, 12, 18, 19]. Indeed, according to our estimations, experimental transmission loss below $2 \mathrm{~dB}$ is achievable for $\mathrm{Ag} / \mathrm{PTFE}$ waveguides provided larger diameters are used. Nevertheless, given the exceptional low absorption of COP at terahertz frequencies, Ag/PTFE HMWs should not outperform $\mathrm{Ag} / \mathrm{COP} \mathrm{HMWs}$ in terms of transmission loss. However, the range of COP tubes with different diameter and size is not yet as extensive as PTFE tubes, which limit the flexibility of the fabrication approach presented.

\section{Conclusion}

We characterize for the first time PTFE-lined hollow metallic waveguides operating at 1.0-2.0 THz experimentally using near-field time-domain measurements and numerically using eigenmode calculations. We report effective quasi-single-mode propagation for the $1.0<\nu<1.6 \mathrm{THz}$ (i.e., $\mathrm{HE}_{11}{ }^{1}$ mode band) with a baseline transmission loss $\sim 20 \mathrm{~dB} / \mathrm{m}\left(\sim 0.046 \mathrm{~cm}^{-1}\right)$ and the multimode propagation for $\nu>1.6 \mathrm{THz}$.

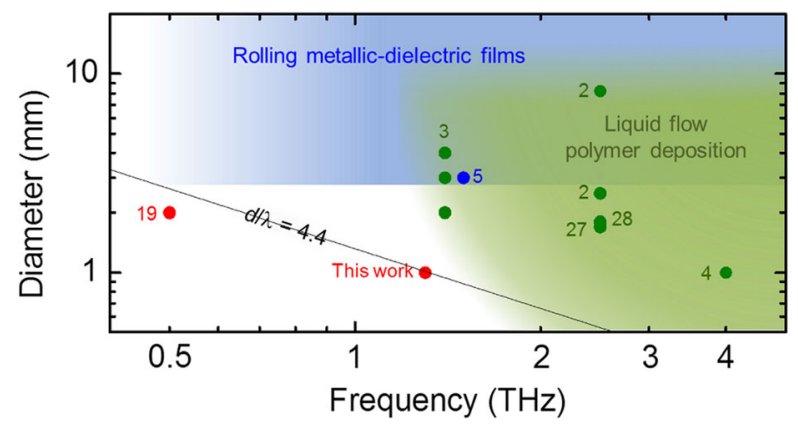

Fig. 9 Diameter-frequency map of the reported dielectric-lined HMWs categorized according to fabrication techniques: mandrel-wiring (blue), dynamic liquid phase (green), and coating of polymer tubes (red). The numbers indicate the references from which the data are taken 
The fabrication approach employed here to produce a quasi-single-mode dielectric-lined hollow waveguide holds promise for the $1.0-2.0 \mathrm{THz}$ range owing to its simplicity (coating commercially available tubes) and because the use of alternative fabrication techniques remains elusive. The proposed approach is universal and can be applied to any other polymer provided the suitable etching agent is used such that a silver film can be effectively deposited. Therefore, it is necessary to investigate other polymers with known lower absorption values in the region of interest such as COP.

Acknowledgments This work was supported in part by the Engineering and Physical Sciences Research Council (grant numbers EP/G033870/1 and EP/J017671/1), and the Royal Society (grant number UF080745). The work of M. Navarro-Cía was supported by Imperial College London through a Junior Research Fellowship.

Conflict of Interest The authors declare that they have no conflict of interest.

\section{Appendix}

Eigenmode numerical analysis

The transmission properties of the Ag-coated PTFE tube are modeled with CST Microwave Studio ${ }^{\mathrm{TM}}$ using a two-dimensional frequency-domain eigenmode analysis [4, 19, 25]. To simplify the calculation, the thickness of the silver film is considered semiinfinite (i.e., the background material outside the PTFE tube is silver). Given that the Ag film thickness is much larger than the skin depth within the spectral region of interest, this simplification does not have any effect in the waveguide modes. An effective dispersive conductivity calculated via the Hammerstad-Jensen approach that takes into account the roughness of the metal is used to model the background material silver. We assume that the roughness of the metal is equal to the measured average RMS roughness of the PTFE outer surface after the etching procedure, but before the deposition of the silver film: $60 \mathrm{~nm}$. The simulated geometry consists then of a 38- $\mu \mathrm{m}$-thick PTFE tube of $1 \mathrm{~mm}$ diameter loss-free air core. The dielectric property of the PTFE is based on tabulated experimental data $[22,23]$. Four times higher dielectric losses are also studied to account for potential fabrication issues and to show the effect of this parameter in the loss spectrum. Given the twofold symmetry of the problem, a vertical electric and a horizontal magnetic mirror planes are applied to consider only a quarter of the waveguide cross-section. An adaptive tetrahedral discretization with maximum edge length of $10 \mu \mathrm{m}$ within the PTFE tube is used to match accurately the circular cross-sectional area of the waveguide.

To study the attenuation due to bending, the commercial software Lumerical MODE Solutions is used in this case. The same material properties as for CST Microwave Studio $^{\mathrm{TM}}$ (nominal PTFE and effective dispersive conductivity for RMS roughness $60 \mathrm{~nm}$ ) are used. Twofold symmetry is no longer valid, and thus, only vertical electric or horizontal magnetic mirror planes are used for the $y z$ - or $x z$-plane of bending case, respectively. A hexahedral mesh $1.3 \mu \mathrm{m} \times 1.3 \mu \mathrm{m}$ is applied in this case. The additional bending loss between the straight waveguide and the waveguide with bending radius $R=50 \mathrm{~mm}$ computed with the Lumerical MODE Solutions is added to the attenuation loss of the straight waveguide calculated with CST Microwave Studio $^{\mathrm{TM}}$ to generate Fig. $5 \mathrm{a}, \mathrm{b}$. 
Open Access This article is distributed under the terms of the Creative Commons Attribution 4.0 International License (http://creativecommons.org/licenses/by/4.0/), which permits unrestricted use, distribution, and reproduction in any medium, provided you give appropriate credit to the original author(s) and the source, provide a link to the Creative Commons license, and indicate if changes were made.

\section{References}

1. O. Mitrofanov, R. James, F. Aníbal Fernández, T. K. Mavrogordatos, J.A. Harrington, IEEE Trans. THz Sci. Tech. 1, 124 (2011)

2. B. Bowden, J. A. Harrington, O. Mitrofanov, Appl. Phys. Lett. 93, 181104-1-3 (2008)

3. P. Doradla, C. S. Joseph, J. Kumar, R. H. Giles, Opt. Express 20, 19176 (2012)

4. M. Navarro-Cía, M.S. Vitiello, C.M. Bledt, J.E. Melzer, J.A. Harrington, O. Mitrofanov, Opt. Express 21, 23748 (2013)

5. Y. Matsuura, E. Takeda, J. Opt. Soc. Am. B 25, 1949 (2008)

6. Y. Yang, M. Mandehgar, D. R. Grischkowsky, IEEE Trans. THz Sci. Tech. 2, 406 (2012)

7. R. Mendis, D. M. Mittleman, Opt. Express 17, 14839 (2009)

8. T.-I. Jeon, D. Grischkowsky, Appl. Phys. Lett. 88, 06113 (2006)

9. Y. Yang, M. Mandehgar, D. Grischkowsky, IEEE T. THz Sci, Tech. 1, 264 (2011)

10. S. E. Miller, A. C. Beck, Proc. IRE 41, 348 (1953)

11. A.A. Kostenko, A.I. Nosich, P. F. Goldsmith, in History of Wireless, ed. By T. K. Sarkar, R. Mailloux, A. A. Oliner, M. Salazar-Palma, D. L. Sengupta (Wiley, Hoboken, New Jersey, 2006), ch. 15, pp. 473-542

12. E.A.J. Marcatili, R.A. Schmeltzer, Bell Syst. Tech. J. 43, 1783 (1964)

13. L. N. Vershinina, Y. N. Kasantsev, V. V. Meriakri,V. V. Shevchenko, in 1st European Microwave Conference, London, UK, 1969, p 251

14. A.I. Goroshko, Y.M. Kuleshov, Radiotekhnika 21, 215 (1972)

15. J.W. Carlin, P. D'Agostino, Bell Syst. Tech. J. 52, 453 (1973)

16. J.W. Carlin, A. Maione, Bell Syst. Tech. J. 52, 487 (1973)

17. R. K. Nubling, J. A. Harrington, Opt. Eng. 37, 2454 (1998)

18. X.-L. Tang, Y.-W. Shi, Y. Matsuura, K. Iwai, M. Miyagi, Opt. Lett. 34, 2231 (2009)

19. J. E. Melzer, M. Navarro-Cía, O. Mitrofanov, J. A. Harrington, Proc. SPIE 8938, 89380I (2014)

20. J. A. Harrington, Infrared fiber optics and their applications, 1st edn. (SPIE, Bellingham, Washington, 2004)

21. R.J. Boyd, W.E. Cohen, W.P. Doran, R.D. Tuminaro, The Bell Syst. Tech. J. 56,1873 (1997)

22. C. Winnerwisser, F. Lewen, H. Helm, Appl. Phys. A 66, 593 (1998)

23. J. Kawamura, S. Paine, and D.C. Papa, in Proceedings of the Seventh International Symposium on Space Terahertz Technology, (National Radio Astronomy Observatory, 1996), p. 349.

24. http://www.zeusinc.com/

25. M. Navarro-Cía, C. M. Bledt, M. S. Vitiello, H. E. Beere, D. A. Ritchie, J. A. Harrington, O. Mitrofanov, J. Opt. Soc. Am. B 30, 127 (2013)

26. R. Mueckstein, M. Navarro-Cía, O. Mitrofanov, Appl. Phys. Lett. 102, 141103 (2013)

27. O. Mitrofanov, T. Tan, P. R. Mark, B. Bowden, J. A. Harrington, Appl. Phys. Lett. 94, 171104 (2009)

28. O. Mitrofanov, J. A. Harrington, Opt. Express 18, 1898 (2010)

29. O. Mitrofanov, M. Lee, J.W.P. Hsu, I. Brener, R. Harel, J.F. Federici, J.D. Wynn, L.N. Pfeiffer, K. W. West, IEEE J. Sel. Top. Quantum Elect. 7, 600 (2001)

30. Y.-S. Jin, G.-J. Kim, S.-G. Jeon, J. Korean Phys. Soc. 49, 513 (2006)

31. P. D. Cunningham, N. N. Valdes, F. A. Vallejo, L. M. Hayden, B. Polishak, X.-H. Zhou, J. Luo, A. K.-Y. Jen, J. C. Williams, R. J. Twieg, J. Appl. Phys. 109, 043505 (2011)

32. Y. Kato, M. Miyagi, IEEE Trans. Microw. Theor. Tech. 40, 679 (1992)

33. M. Miyagi, S. Kawakami, J. Lightwave Tech. LT-2, 116 (1984)

34. Y. Kato, M. Miyagi, IEEE Trans. Microw. Theor. Tech. 42, 2336 (1994)

35. http://vadiodes.com/VDI/pdf/waveguidechart200908.pdf 